\title{
On strategy for determining displacement from perturbed phase of self mixing interferometry when parameters are unknown
}

\author{
Junaid Iqbal Khan \\ School of Electrical Engineering and Computer Science \\ national University of Sciences and Technology \\ Islamabad,Pakistan \\ jkhan.bee15seecs@seecs.edu.pk
}

\begin{abstract}
While self-mixing interferometry(SMI) has proven to be suitable for displacement measurement and other sensing applications, its characteristic self-mixing signal shape is strongly governed by the non-linear phase equation which forms relation between perturbed and unperturbed phase of self-mixing laser. Therefore, while it is desirable for robust estimation of displacement of moving target, the algorithms to achieve this must have an objective strategy that can be achieved by understanding the characteristic of extracting knowledge of the perturbed phase from the unperturbed phase. Therefore, it has been proved and shown that such a strategy must not involve sole methods where the perturbed phase is a continuous function of the unperturbed phase (e.g: Taylor series or fixed-point methods) or through successive displacements (e.g: variations of Gauss-Seidel method). The subset of this strategy is to perform spectral filtering of the perturbed phase followed by perturbative or homotopic deformation. A less computationally expensive approach of this strategy is adopted to achieve displacement with a mean error of $62.2 \mathrm{~nm}$ covering all feedback regimes, when the coupling factor ' $C$ ' is unknown.
\end{abstract}

Index Terms-Self mixing interferometery,phase equation, $L_{2}$ triangular ineqaulity, Riesz-Thorin theorem, convolution theorem,sine half and double angle identity

\section{INTRODUCTION}

Laser diode(LD) based Self-Mixing (SM) or optical feedback interferometry is an attractive sensing scheme due to the compact, self-aligned, and low-cost nature of the SM sensor [11]. A basis process of self mixing

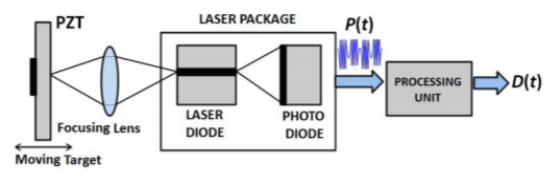

Fig. 1. Schematic diagram of self-mixing laser sensor for displacement measurement requiring only a laser package and a lens. A piezoelectric transducer (PZT) has been used as remote target. Variations in the optical output power $\mathrm{P}(\mathrm{t})$ are processed by a computing unit in order to retrieve the target displacement $\mathrm{D}(\mathrm{t})$

phenomenon for displacement measurement is shown in figure(1). An SM sensor is much simpler than conventional interferometers because many optical elements,such as the beam-splitter, reference mirror and external photo-detector are not required.Thus, with a simply constructed opto-electronic system, smart laser non-contact sensors have been developed using SM [10] with high precision, [3]. 2]. The model of optical feedback by Lang and Kobayashi [6], shows that displacement retrieval from a weak feedback regime SM signal was achieved by unwrapping the laser feedback phase. It used pre-calibrated values of two fundamental SM parameters: line-width enhancement factor $\alpha$ and optical feedback coupling factor $C$. The method is claimed to have measurement error of $<50 \mathrm{~nm}$ in displacement reconstruction for a laser wavelength of $673 \mathrm{~nm}$, resulting in an accuracy of about $\frac{\lambda}{13}$. Further improvement was achieved by an auto-adaptive SM retrieval algorithm, called as the phase unwrapping method(PUM), for moderate feedback regime [2]. It consists of two major steps: 1) rough estimation of laser phase under feedback $\phi_{t}$ and 2) joint estimation of $\mathrm{C}, \theta=\arctan (\alpha)+\phi_{o}$, and displacement $\mathrm{D}(\mathrm{t})$, where $\phi_{o}$ represents the initial laser phase under feedback. The proposed method increases the accuracy of the SM sensor to $\frac{\lambda}{16}$. The root of this research was tackled in [8], where the behavioral model of a self-mixing laser diode sensor was presented to simplify the solutions to non-linear self interferometric phase equation and thus modelling the basis of all algorithms using piecewise analysis on cases of $\mathrm{C}$. Moreover, in [11], the TFSP method was introduced where perturbed phase was estimated from unperturbed phase thus removing the need for estimation of parameters of phase equation, using Fourier series but to my understanding, the logic of generally connecting Fourier coefficients of perturbed phase and unperturbed phase remained unproven. Therefore analysis has been performed on this paper to rigorously deduce the spectral property of the perturbed phase as compared to unperturbed phase, along with providing theoretical background on deriving them in time domain.

\section{Main theory of Self Mixing Interferometry}

Laser beam is generated in the optical cavity of a laser diode (LD) and a portion of the laser beam is back-scattered 
from the target and re-enters the active laser cavity. Let $\mathrm{D}(\mathrm{t})$ represent the instantaneous distance between the LD driven by a constant injection current and a remote surface which backscatters a small amount of optical power back into the LD cavity [8]. When this optical feedback phenomenon occurs, the laser wavelength is no longer the constant $\lambda_{o}$ but is slightly modified and becomes a function of time $\lambda_{f}(t)$ when $\mathrm{D}(\mathrm{t})$ varies. The wavelength fluctuations can be found by solving the phase equation,

$$
x_{o}(t)=x_{f}(t)+C \sin \left(x_{f}(t)+\arctan (\alpha)\right)
$$

$x_{o}$ and $x_{f}$ are referred as perturbed and unperturbed phase respectively. $\mathrm{C}$ is the optical feedback coupling factor and $\alpha$ is the line enhancement factor. $x_{o}$ and $x_{f}$ can be represented as,

$$
\begin{aligned}
& x_{o}(t)=2 \pi \nu_{o}(t) \tau(t) \\
& x_{f}(t)=2 \pi \nu_{f}(t) \tau(t)
\end{aligned}
$$

where $\tau(t)=2 D(t) / c$ is the round trip time, with $\mathrm{c}$ as speed of light. $\nu_{f}(t)$ and $\nu_{o}(t)$ represents optical frequencies with and without feedback. As $\mathrm{C}$ increases from zero, the laser operates into five different regimes.Generally, (self mixing)SM sensing is performed under weak feedback regime $(C<1)$, moderate feedback regime $(1<C<4.6)$, or strong feedback regime $(C>4.6)$. However, moderate feedback regime is usually preferred over others as the simple saw-tooth shaped SM fringes belonging to such a regime [1] intrinsically provide motion direction indication and require simplified SM fringe detection processing (while both tasks are difficult to achieve for weak feedback regime). Laser feedback output optical power(LDOOP) $\mathrm{P}(\mathrm{t})$ depends on the SM phenomenon and written as $P(t)=P_{o}\left[1+m \cos \left(x_{f}(t)\right)\right]$ where $P_{o}$ is the power emitted by the free running state LD and $\mathrm{m}$ is the modulation index.From above, perturbed phase $x_{f}$ can be extracted, which can be used to calculate unperturbed phase $x_{o}$ and then displacement $\mathrm{D}(\mathrm{t})$ of the vibrating target by $D(t)=\frac{\lambda_{o} x_{o}}{4 \pi}$

\section{ANALYSIS}

A. Impossibility of derivation of perturbed phase as continuous function of unperturbed phase from SMI phase equation

Consider the SMI phase equation,

$$
x_{o}=x_{f}+C \sin \left(x_{f}+\arctan (\alpha)\right)
$$

Putting $\theta=\arctan (\alpha)$,

$$
x_{f}-x_{o}=-C \sin \left(x_{f}+\theta\right)
$$

Suppose that $x_{o}$ is a continuous function of $x_{f}, x_{o}=G\left(x_{f}\right)$, whose continuous inverse exists, then $x_{f}=G^{-1}\left(x_{o}\right)=$ $H\left(x_{o}\right)$. If after following steps solutions obtained are not extraneous,then our assumption is correct. using sine double angle identity,

$$
\begin{gathered}
\sin (2 x)=2 \sin (x) \cos (x) \\
\sin (x)=2 \sin (x / 2) \cos (x / 2)
\end{gathered}
$$
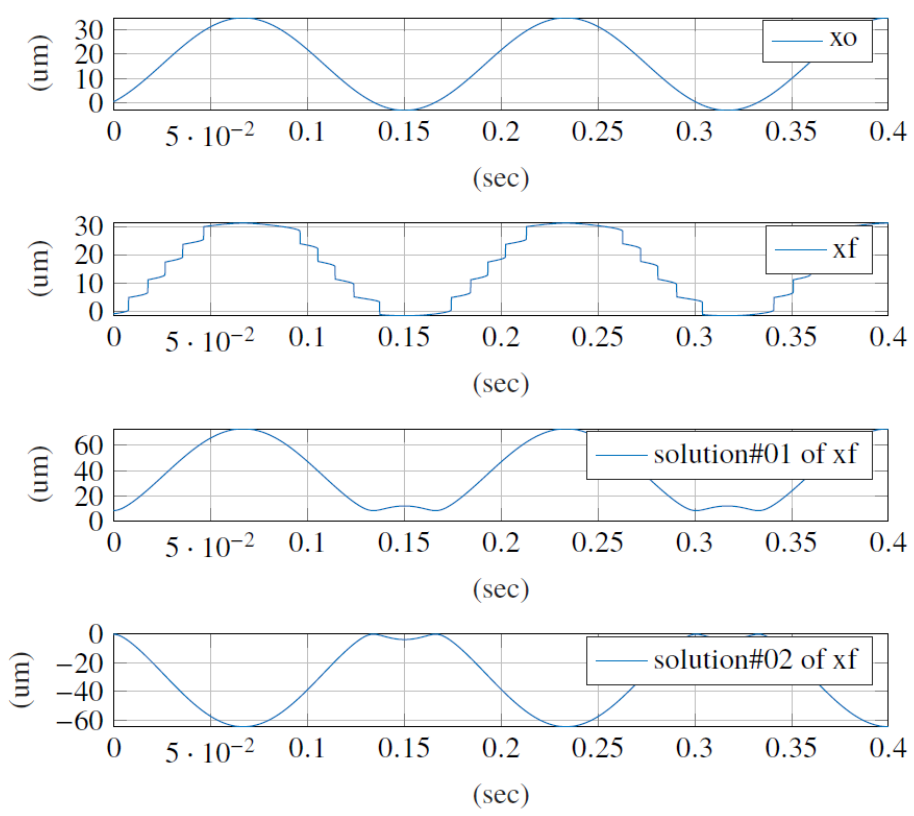

Fig. 2. Comparison of errors for estimated perturbed phase $x_{f}$ after 7,10 and 13 substitutions for $6 \mathrm{~Hz}$ displacement at $\mathrm{C}=4, \alpha=5$

$$
\sin (x)=2 \sin (x / 2) \sin (\pi / 2-x / 2)
$$

we get,

$$
x_{f}-x_{o}=-2 C \sin \left(\frac{x_{f}+\theta}{2}\right) \sin \left(\frac{\pi}{2}-\frac{x_{f}+\theta}{2}\right)
$$

Bringing the arguments of sine to form of $(f+\theta)$

$$
x_{f}-x_{o}=-2 C \sin \left(\frac{x_{f}-\theta}{2}+\theta\right) \sin \left(\frac{\pi}{2}-\frac{x_{f}}{2}-\frac{3 \theta}{2}+\theta\right)
$$

Using the original phase equation, we can make substitutions as,

$$
x_{f}-x_{o}=-2 C\left(\frac{x_{o}-\frac{x_{f}-\theta}{2}}{C}\right)\left(\frac{x_{o}-\frac{\pi-x_{f}-3 \theta}{2}}{C}\right)
$$

The above forms a quadratic equation, whose solutions of $x_{f}$ in terms of $x_{o}$ appear as,

$x_{f}=\frac{1}{2}\left(2 C+\pi-2 \theta \pm \sqrt{4 C^{2}+4 C \pi+\pi^{2}-8 C \theta-8 \pi \theta+16 \theta^{2}-8 C x_{o}-8 \pi x_{o}+32 \theta x_{o}+16 x_{o}^{2}}\right)$

Firstly, these solutions from equation (7) are extraneous as, when put back in equation (2), we do not get any feasible relation, as no identity of sine function acting on under-root leads to an algebraic equation. Therefore, all analytic solutions are extraneous.

On simulating results by figure(2), the simulated perturbed phase does not match theoretical perturbed phase solutions. Therefore, all the solutions derived are extraneous and thus no continuous mapping from $x_{o}$ to $x_{f}$ can satisfy phase equation, Also, we can deduce that any method (e.g: fixed-point iterations, dynamic programming or Taylor series method, as long as they are based on continuous mapping) can not solely estimate displacement when we only know perturbed phase (without knowledge of parameters). 
B. Impossibility of achieving perturbed phase from unperturbed phase through successive displacements or substitutions from SMI phase equation

Suppose,that we can derive perturbed phase $x_{f}$ from phase equation (3) through successive displacements or substitutions.
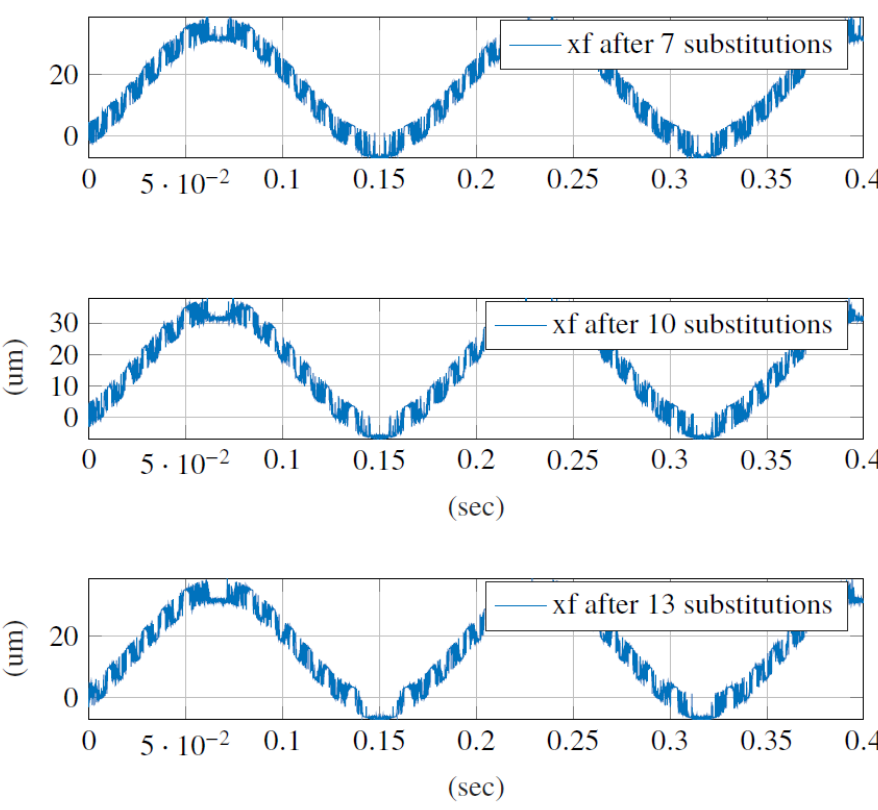

Fig. 3. Comparison of estimated perturbed phase $x_{f}$ after 7,10 and 13 substitutions for $6 \mathrm{~Hz}$ displacement at $\mathrm{C}=4, \alpha=5$
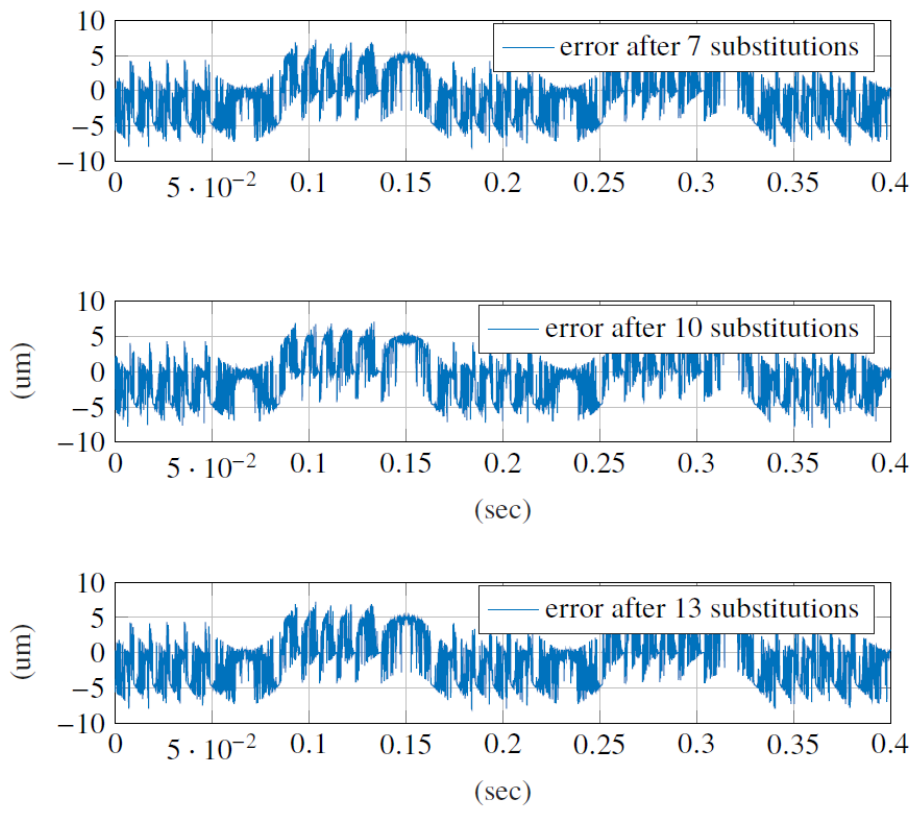

Fig. 4. Comparison of errors for estimated perturbed phase $x_{f}$ after 7,10 and 13 substitutions for $6 \mathrm{~Hz}$ displacement at $\mathrm{C}=4, \alpha=5$

Then substituting $x_{f}$ back into the right hand side of (3),and thus removing $x_{f}$ on right side, and continuing such displacements, we get

$$
x_{f}=x_{o}-C \sin \left(x_{o}+\theta-C \sin \left(x_{o}+\theta-C \sin \left(x_{o}+\theta+\ldots\right)\right)\right)
$$

Lets denote,

$$
S_{x_{o}, C, \theta} \rrbracket=-C \sin \left(x_{o}+\theta+\llbracket\right)
$$

then

$$
x_{f}=x_{o}+S_{x o, C, \theta}\left[S_{x o, C, \theta}[\ldots]\right]
$$

Suppose, in order to approximate $x_{f}$, we could replace nth recursion $S_{x_{o}, C, \theta}[\ldots]_{n}$ by a constant ' $k$ ', then as 'n' approaches infinity,then,

$$
\begin{gathered}
\lim _{n \rightarrow \infty}\left(\left[x_{f}\right]_{n}-\left[x_{f}\right]_{n+1}\right) \\
\lim _{n \rightarrow \infty}(k)=\lim _{n \rightarrow \infty}\left(-C \sin \left(x_{o}+\theta+\left[S_{x o, C, \theta}[\ldots]\right]_{n+2}\right)\right)
\end{gathered}
$$

since,

$$
x=A * \sin (x+t) \ldots . \forall t \neq 0
$$

has no solutions for $\mathrm{x}$,therefore (12) is invalid and (8) does not converge to $x_{f}$. For purpose of demonstration of result, note that,

$$
0 \leq\left|S_{x o, C, \theta}[]\right| \leq C
$$

For testing the result, we can just halt the substitutions by replacing $S_{x o, C, \theta}[]$ by $\frac{C+0}{2}=\frac{C}{2}$. On simulating the results, as shown in figure (3) and (4), simulated perturbed and theoretical perturbed phase do not appear the same at all because the error between successive recursive operations appear same. Thus equation (8) is an extraneous solution to equation (2) and the assumption of deriving it is wrong.

\section{Nature of perturbed and unperturbed phase in frequency} domain

We are going to use norm $\left(L_{2}\right)$,in order to exploit the normmodulus relationship,

$$
\epsilon=-C \sin \left(x_{f}+\arctan (\alpha)\right)
$$

Taking Fourier Transform on both sides and letting $\theta=$ $\arctan (\alpha)$

$$
\begin{aligned}
& F[\epsilon]=-C F[\sin (x f+\arctan (\alpha))] \\
& F[\epsilon]=-C \sum_{k \geq 0}(-1)^{k} \frac{F\left[\left(x_{f}+\theta\right)^{2 k+1}\right]}{(2 k+1) !}
\end{aligned}
$$

4 Using Convolution theorem,

$$
F[\epsilon]=-C \sum_{k \geq 0}(-1)^{k \frac{*_{1}^{2 k+1} F\left[\left(x_{f}+\theta\right)\right]}{(2 k+1) !}}
$$

Taking norm $\left(L_{2}\right)$ on both sides,

$$
\begin{gathered}
\|F[\epsilon]\|=C \| \sum_{k \geq 0}(-1)^{k \frac{*_{1}^{k k+1} F\left[\left(x_{f}+\theta\right)\right]}{(2 k+1) !} \|} \\
\|F[\epsilon]\|=C\left\|\sum_{k \in \text { even }} \frac{*_{1}^{2 k+1} F\left[\left(x_{f}+\theta\right)\right]}{(2 k+1) !}-\sum_{k \in \text { odd }} \frac{*_{1}^{2 k+1} F\left[\left(x_{f}+\theta\right)\right]}{(2 k+1) !}\right\| \\
\text { Under frame of triangular inequality }
\end{gathered}
$$

$$
\|a\|-\|b\| \leq\|a+b\| \leq\|a\|+\|b\|
$$

and Riesz-Throin theorem [5],for $\mathrm{p} \geq 1$,

$$
\|f * f\|_{p} \leq\|f\|_{1} \cdot\|f\|_{p}
$$


which implies,

$$
\left\|*_{1}^{2 k+1} f\right\|_{2} \leq\left(\|f\|_{1}\right)^{2 k+1}
$$

Representing, \|\|$_{2}$ as \|\| ,

$$
\begin{aligned}
& \|F[\epsilon]\|=C\left\|\sum_{k \in \text { even }} \frac{*_{1}^{2 k+1} F\left[\left(x_{f}+\theta\right)\right]}{(2 k+1) !}-\sum_{k \in \text { odd }} \frac{*_{1}^{2 k+1} F\left[\left(x_{f}+\theta\right)\right]}{(2 k+1) !}\right\| \longrightarrow(a)
\end{aligned}
$$

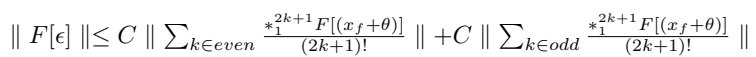

$$
\begin{aligned}
& \|F[\epsilon]\| \leq C \sum_{k \in \text { even }} \frac{\left\|*_{1}^{2 k+1} F\left[\left(x_{f}+\theta\right)\right]\right\|}{(2 k+1) !}+C \sum_{k \in \text { odd }} \frac{\left\|*_{1}^{2 k+1} F\left[\left(x_{f}+\theta\right)\right]\right\|}{(2 k+1) !} \\
& \|F[\epsilon]\| \leq C \sum_{k \geq 0} \frac{\left(\left\|F\left[x_{f}+\theta\right]\right\|_{1}\right)^{2 k+1}}{(2 k+1) !}
\end{aligned}
$$

Since,

$$
\sinh (x)=\sum_{k>0} \frac{x^{2 k+1}}{(2 k+1) !}
$$

therefore,

$$
\|F[\epsilon]\| \leq C \sinh \left\|F\left[x_{f}+\theta\right]\right\|_{1}
$$

Using (25),

$$
\|F[\epsilon]\|=C\left\|\sum_{k \in e v e n} \frac{*_{1}^{2 k+1} F\left[\left(x_{f}+\theta\right)\right]}{(2 k+1) !}-\sum_{k \in \text { odd }} \frac{*_{1}^{2 k+1} F\left[\left(x_{f} f \theta\right)\right]}{(2 k+1) !}\right\| \longrightarrow(a)
$$

Applying triangular inequality again,

$$
\|F[\epsilon]\| \geq C\left\|\sum_{k \in \text { even }} \frac{*_{1}^{2 k+1} F\left[\left(x_{f}+\theta\right)\right]}{(2 k+1) !}\right\|-C\left\|\sum_{k \in \text { odd }} \frac{*_{1}^{*_{1}^{k+1}} F\left[\left(x_{f}+\theta\right)\right]}{(2 k+1) !}\right\|
$$

If the second normed sum is increased the inequality will surely retain as the overall result will be lesser if the first normed sum is fixed. So manipulating second normed sum,

$$
C\left\|\sum_{k \in \text { odd }} \frac{*_{1}^{2 k+1} F\left[\left(x_{f}+\theta\right)\right]}{(2 k+1) !}\right\| \leq C \sum_{k \in \text { odd }} \frac{\left(\left\|F\left[x_{f}+\theta\right]\right\|_{1}\right)^{2 k+1}}{(2 k+1) !} \leq C \sum_{k \geq 0} \frac{\left(\left\|F\left[x_{f}+\theta\right]\right\|_{1}\right)^{2 k+1}}{(2 k+1) !}
$$

and,

$$
C \sum_{k \geq 0} \frac{\left(\left\|F\left[x_{f}+\theta\right]\right\|_{1}\right)^{2 k+1}}{(2 k+1) !}=C \cdot \sinh \left\|F\left[x_{f}+\theta\right]\right\|_{1}
$$

Let

$$
f\left(x_{f}, \theta\right)=\left\|\sum_{k \in \text { even }} \frac{{ }_{*}^{2 k+1} F\left[\left(x_{f}+\theta\right)\right]}{(2 k+1) !}\right\|
$$

by using again triangular inequalities, just by add and subtracting suitable terms for higher bound and truncating at $\mathrm{k}=0$ for the lower bound, we can find

$$
\left\|F\left[x_{f}+\theta\right]\right\| \leq f\left(x_{f}, \theta\right) \leq \frac{\sin \left(\left\|F\left[x_{f}+\theta\right]\right\|_{1}\right)+\sinh \left(\left\|F\left[x_{f}+\theta\right]\right\|_{1}\right)}{2}
$$

Combining results,

$$
\|F[\epsilon]\| \geq C f\left(x_{f}, \theta\right)-C \sinh \left(\left\|F\left[x_{f}+\theta\right]\right\|_{1}\right)
$$

Combining (30),(36) and (37),

$$
C f\left(x_{f}, \theta\right)-C \sinh \left(\left\|F\left[x_{f}+\theta\right]\right\|_{1}\right) \leq\|F[\epsilon]\| \leq C \sinh \left(\left\|F\left[x_{f}+\theta\right]\right\|_{1}\right)
$$

where,

$$
\left\|F\left[x_{f}+\theta\right]\right\| \leq f\left(x_{f}, \theta\right) \leq \frac{\sin \left(\left\|F\left[x_{f}+\theta\right]\right\|_{1}\right)+\sinh \left(\left\|F\left[x_{f}+\theta\right]\right\|_{1}\right)}{2}
$$

Firstly, from equation(3), $x_{o}-x_{f}=C \sin \left(x_{f}+\theta\right)$ Since sine is bounded function, therefore, $x_{F}$ acts as a cover around $x_{o}$ with maximum variation of $C$.
Moreover,for the purpose of analysis, lets define $\mu_{\tau}(\omega)$,defined over $\omega \geq 0$ as

$$
\mu_{\tau}(\omega)=1 \text { iff } \omega \leq \tau, \text { otherwise } 0
$$

then,

$$
\begin{gathered}
F_{\tau}\left[x_{o}(t)\right]=F\left[x_{o}(t)\right] \mu_{\tau}(\omega) \\
F_{\tau}\left[x_{f}(t)\right]=F\left[x_{f}(t)\right] \mu_{\tau}(\omega) \\
F_{\tau}[\epsilon(t)]=F[\epsilon(t)] \mu_{\tau}(\omega)
\end{gathered}
$$

Consider, $x_{o}(t)$ as sum of finite $\mathrm{N}$ sinusoids as,

$$
x_{o}(t)=\sum_{n \geq 0}^{N} a_{n} \cos \left(\omega_{n}(t)\right)+b_{n} \sin \left(\omega_{n}(t)\right)
$$

Taking absolute of Fourier transform,we get,

$\left|F\left[x_{o}(t)\right]\right|=\sqrt{\frac{\pi}{2}} \sum_{n \geq 0}^{N}\left(a_{n}+b_{n}\right)\left(\delta\left(\omega-\omega_{n}\right)+\delta\left(\omega-\omega_{n}\right)\right)+\left(a_{m}-b_{n}\right)\left(\delta\left(\omega-\omega_{n}\right)-\delta\left(\omega+\omega_{n}\right)\right)$

Please note such function forms an almost zero everywhere for domain $\omega \geq 0$. When $\tau$ is allowed to vary from left to right of interval, $\left\|F_{\tau}\left[x_{o}(t)\right]\right\|=\int_{0}^{\tau}\left|F_{\tau}\left(x_{o}\right)\right|^{2}$ increases discontinuously but rate is extremely low due to almost zero every where behaviour. On the other hand,from (38), $\|$ $F_{\tau}\left[x_{o}(t)\right] \|$ increases rapidly and continuously by virtue of $\sinh \left(\left\|F_{\tau}\left[x_{f}(t)\right]+\frac{\theta}{\iota \omega}\right\|\right)$. This also allows greater magnitudes along higher frequencies. Therefore, from above, one can make a plausible inference that, in frequency domain, $x_{f}$ acts as noisy version (coverage of magnitudes over more frequency domain)of $x_{o}$. This also gives indication that $\left|F\left(x_{f}-x_{o}\right)\right|$ has no information of $x_{o}$. This is only possible, if $F\left(x_{f}\right)$ act as modified version of $F\left(x_{o}\right)$, then $F\left(x_{f}-x_{o}\right)=F\left(x_{f}\right)-F\left(x_{o}\right)$ would algebraically cancel out $F\left(x_{o}\right)$,removing information of $x_{o}$ to some extent,in frequency domain.

$$
F\left[x_{f}\right](\omega)=F\left[x_{o}\right](\omega)+T(\omega)
$$

where, as per our assumption, $F\left[x_{o}\right](\omega)$ is almost zero everywhere (with some non zero elements at finite frequencies) and but $T(\omega)$ forms a continuous function with coverage over larger frequency domain and $F$. Thus, the extraction of $x_{o}$ from $x_{f}$ in frequency domain, becomes a spectral filtering process followed by minimizing contribution of $T(\omega)$ superimposed on $F\left[x_{o}\right](\omega)$.This can be achieved by following some perturbative or homotopical deformation methods such that dynamics of equation(1) become preserved, to tackle the problem of superposition. It is to be noted that this is not simple as ordinary filtering process and error is not random and has structure. This approach has been used in TFSP method [11] to estimate $x_{o}$ from $x_{f}$ without knowledge of $\mathrm{C}$ and $\alpha$ by simply putting a low pass filter which is an incomplete procedure. 


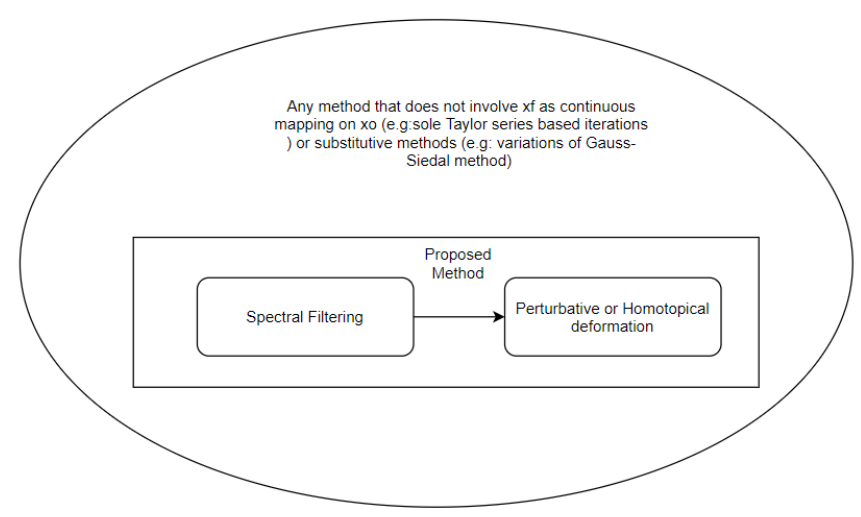

Fig. 5. Venn diagram showing strategy for determination of displacement form sole knowledge of perturbed phase

\section{Algorithm Design}

Since, perturbed phase $x_{f}$ cannot be represented as a continuous function of unperturbed phase $x_{o}$, therefore methods for determination of displacement cannot involve sole iterative calculus bases methods or just any ingenious substitution method (e.g: variations of Gauss-Siedal method). For example, they cannot just rely on Taylor expansion to just estimate displacement from $x_{f}$ without going into knowledge of parameters $\mathrm{C}$ and $\alpha$. Moreover, the TFSP method approach [11] has been corrected and proven that moving from perturbed phase to displacement becomes spectral filtering in the frequency domain, followed by perturbative or homotopical deformation in time domain. This can be represented as a Venn diagram as shown in figure (5). Linewidth enhancement factor $(\alpha)$ is most of the time known and even practically it usually its arctangent is taken as $\frac{\pi}{2}$. We can form a straight line homotopy between filtered perturbed phase $x_{o e}$ and $x_{o}$ as equation(40), parameterised by $s \in[0,1]$ in such a way that when s moves from 0 to 1 , xoe deforms into $x_{o}$.

$$
\zeta(s, t)=(1-s) x_{o e}(t)+s x_{o}(t)
$$

For purpose of exploiting the strategy in simple manner, lets assume $\alpha$ is known. We define corresponding ' $\mathrm{C}$ ' function as

$$
C=\frac{x_{o r}-x_{f}}{\sin \left(x_{f}+\arctan (\alpha)\right)}
$$

where, 'C' deforms from variable function to some constant function as 's' moves from 0 to 1 .

There can be multiple strategies for deformation of filtered signal and such methods can be computationally expensive, a simple and less computationally intensive one is, that can be exploited will be translation (its reason is mentioned in Remark).In the $\mathrm{x}-\mathrm{y}$ plane, the filtered phase is translated to some time constant $\tau$ in such a way, the rest of the variations are supposed to be acting along the y-axis. Then along the y-axis, we make a deduction, instead of practically deforming to get our desired displacement. Suppose that the final form of $\mathrm{C}$ is written as,

$$
C=\lim _{\epsilon \rightarrow 0}\left(\epsilon t+C_{o}\right)
$$

where $C_{o}$ is our desired C. Now this equation, as linear equation is resultant from,

$$
\frac{\delta}{\delta s} \int_{t_{1}}^{t_{2}} L=0
$$

where L satisfies equation (43) [7] by Euler-Lagrange equation as

$$
\frac{\partial L}{\partial s}=\frac{d}{d t} \frac{\partial L}{\partial s^{\prime}}
$$

In view of (42), $\mathrm{L}$ would equal to, in combination with (43) and (44) as,

$$
L=\sqrt{1+\left(\frac{d C}{d t}\right)^{2}}
$$

Let, $A(s, t)=\int_{t_{1}}^{t_{2}} L d t$,then expanding $\mathrm{A}(\mathrm{s}, \mathrm{t})$ on ' $\mathrm{t}$ ' as Taylor series to first order with parameter ' $h$ ' such that $h$ is very small.

$$
A(s, t)=A(s, t-h)+h \frac{d A(s, t-h)}{d t}
$$

Putting in equation (43), we get,

$$
\frac{\delta}{\delta s}\left(A(s, t-h)+h \frac{d A(s, t-h)}{d t}\right)=0
$$

Since,(43) is time invariant as time acts as dummy variable for it to hold, therefore $\frac{\delta}{\delta s} A(s, t-h)=0$, and thus substituting $t-h=u$,

$$
\begin{gathered}
h \frac{\delta}{\delta s}\left(\frac{d A(s, u)}{d u}\right)=0 \\
\frac{\delta}{\delta s}\left(\frac{d A(s, u)}{d u}\right)=0
\end{gathered}
$$

Equation (49) is an important result for which there can be ingenious implications.Inductively, by increase $\mathrm{h}$ by small amounts and creating higher order expansions, we can prove that for $n \in \aleph$,

$$
\frac{\delta}{\delta s} \frac{d^{n}}{d u^{n}} A(s, u)=0
$$

We can make a deduction from equation (50) (invariant along s-domain) that since nth-differential operator tries to relate orders of neighborhoods of t-domain to the current point, the fact that equation (50) is satisfied means, the if burden is extremized at one point of $t$, it is also extremized in its neighborhoods and there is equidistribution of deformation burden along t-domain. Now from (47) and substituting $\mathrm{u}=\mathrm{t}-\mathrm{h}$,

$$
\begin{aligned}
& h \frac{\delta}{\delta s} \frac{d}{d u} A(s, u)=-\frac{\delta}{\delta s} A(s, u) \\
& \frac{\delta}{\delta s \frac{d}{d u} A(s, u)}=-\frac{\frac{\delta}{\delta s} A(s, u)}{h}
\end{aligned}
$$

For $s_{o}$-neighbourhood of $\mathrm{s}$ (where equation (43) holds), $\frac{\delta}{\delta s} A(s, u) \rightarrow \zeta_{s_{o}}(t)$, such that $\left|\zeta_{s_{o}}(t)\right| \rightarrow 0$ as $s_{o} \rightarrow 0$, then equation (52) becomes,

$$
\frac{\delta}{\delta s} \frac{d}{d u} A(s, u)=-\frac{\zeta_{s_{o}}(t)}{h}
$$

While deducing from s-domain, while observing along tdomain,from definition of $\zeta_{s_{o}}(t)$, when $\zeta_{s_{o}}(t)>0$, variation $A(s, t)$ was initially greater quantity and deforms to smaller quantity. Similarly, when $\zeta_{s_{o}}(t)<0$, variation $A(s, t)$ was smaller and deforms to larger quantity, t-point wise. But (5) represents that, scenario for $\frac{\delta}{\delta s} \frac{d}{d u} A(s, u)$ is opposite to $\zeta_{s_{o}}(t)>0$, and thus the deformation acts along a curve in such a way that there is equal burden along a curve, and thus if a curve corresponding to (1) is deforming to $\mathrm{C}$, then there 


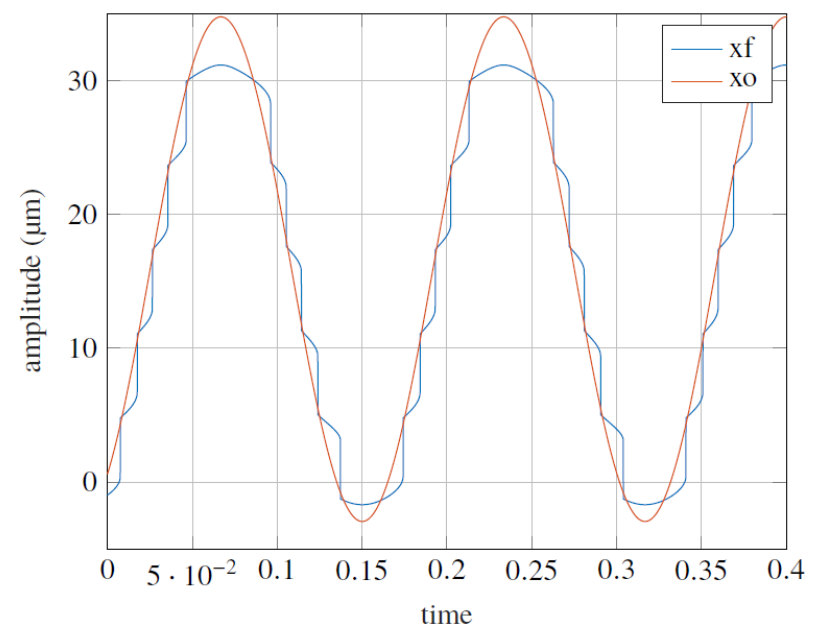

Fig. 6. Comparison of $x_{f}$ (blue) and $x_{o}$ (red) at $6 \mathrm{~Hz}$ for $\mathrm{C}=4, \alpha=5$

would be fixed point along that curve where value would be $\int_{t_{1}}^{t_{2}} C d t$, which will remain fixed point along trajectory along $\mathrm{s}$,till it reaches the extrema, where equation(43) holds and thus, $\int_{t_{1}}^{t_{2}} C d t=C_{o}$.

Now, practically when dealing with discrete form of continuous function, $\int_{t_{1}}^{t_{2}} C d t$ cannot be just translated as arithmetic mean, because then we would be destroying the continuous structure of the original function. Instead, if we can analytically continue along discrete beads, we get the original continuous function back, and thus the best choice is to use the median of discrete ' $C$ ' function which would be our desired parameter of SMI phase equation. Therefore, we can summarise this as the following algorithm:

Step 1: Filter original perturbed phase $x_{f}[n]$ of some length L by some smoothing filter (e.g: Savitzky-Golay filter) to get $x_{o e}[n]$.

Step 2: Choose $\eta$ as error threshold, $k \in \aleph$ starting from 0 and a metric $\xi=\operatorname{mean}\left(\left|x_{o e}[n]-D\left(x_{o e}[n]\right)\right|\right)$, where $x_{o e}[n]$ is our current estimated phase after filtering, and $D\left(x_{o e}[n]\right)$ is resultant of smoothing filter on $x_{o e}[n]$.

Step 3: Extract $\mathrm{k}$ to $\mathrm{L}$ part of $x_{o e}[n]$ as new

Step 4: Substitute vectors in,

$$
C[n]=\frac{x_{o e}[n]-x_{f}[n]}{\sin \left(x_{f}[n]+\arctan (\alpha)\right)}
$$

Find $C_{o}=\operatorname{median}(C[n])$, determine $x_{o e}[n]=x_{f}+$ $C_{o} \sin \left(x_{f}+\arctan (\alpha)\right)$.

Step 5: If $\xi \geq \eta$, repeat step (3) and (4), otherwise $D\left(x_{o e}[n]\right)$ is our desired unperturbed phase.Then $D(n)=\frac{\lambda_{o} D\left(x_{o e}[n]\right)}{4 \pi}$

1) Remark: The idea of translation comes from the fact that moving along any trajectory can be decomposed into moving along the $\mathrm{x}$-axis and then along the $\mathrm{y}$-axis by Pythagoras theorem, and so will be the same for deformation. Another reason is that while we are fitting a function through steps of $x_{f}$ to get filtered output, originally $x_{o}$ covers the $x_{f}$ as just a way that it is tangent to the step corners of $x_{o}$ from above, when $x_{o}$ is increasing, while it is tangent to steps corners from below when $x_{o}$ is decreasing, as shown in figure (6).

\section{Simulation Results}
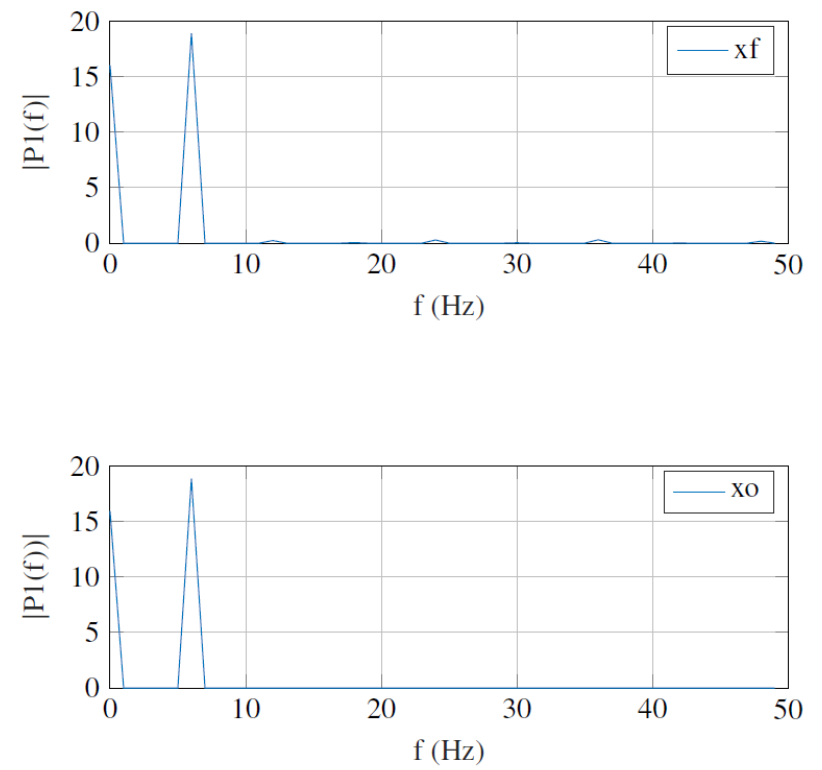

Fig. 7. First plot shows one sided Fourier transform of perturbed phase $x_{f}$ and second plot shows one sided Fourier transform of unperturbed phase $x_{o}$ with magnitude of top peaks at $6 \mathrm{~Hz}$ as 17.75 and 18.84 respectively for $\mathrm{C}=4, \alpha=5$

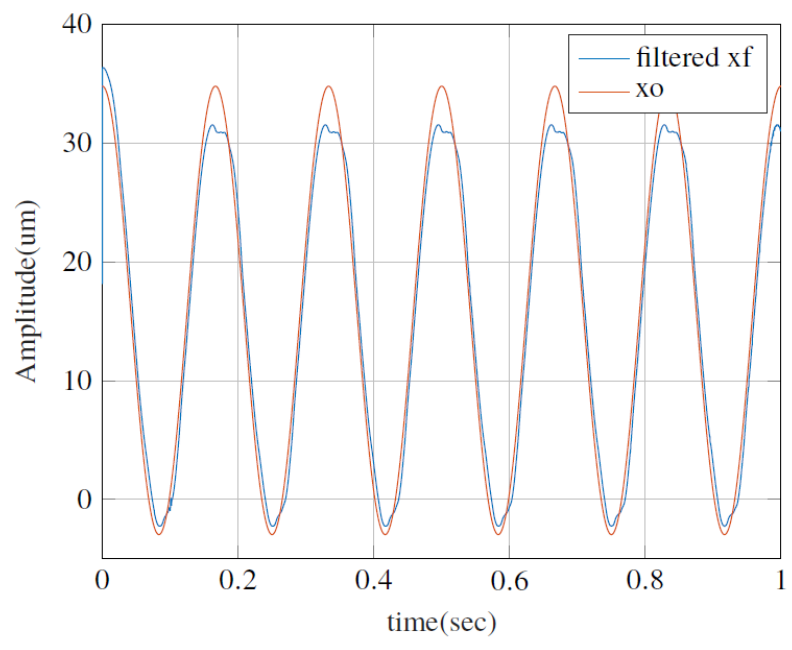

Fig. 8. Comparison of wavelet denoised perturbed phase $x_{f}$ at 7 th level (blue) and unperturbed phase $x_{o}$ (red) for $6 \mathrm{~Hz}$ displacement for $\mathrm{C}=4, \alpha=5$

For purpose of analysis, lets assume $\alpha$ is known. Equation(41) is parameterised by $s \in[0,1]$ in such a way that when s moves from 0 to 1 , then We can translate the concept of spectral processing, as discussed, to be a denoising process based on Fourier norm manipulations, from where we deduced that Fourier transform of $x_{f}$ acts as a cover over Fourier transform of $x_{o}$.It is obvious from the figure (7), that Fourier 


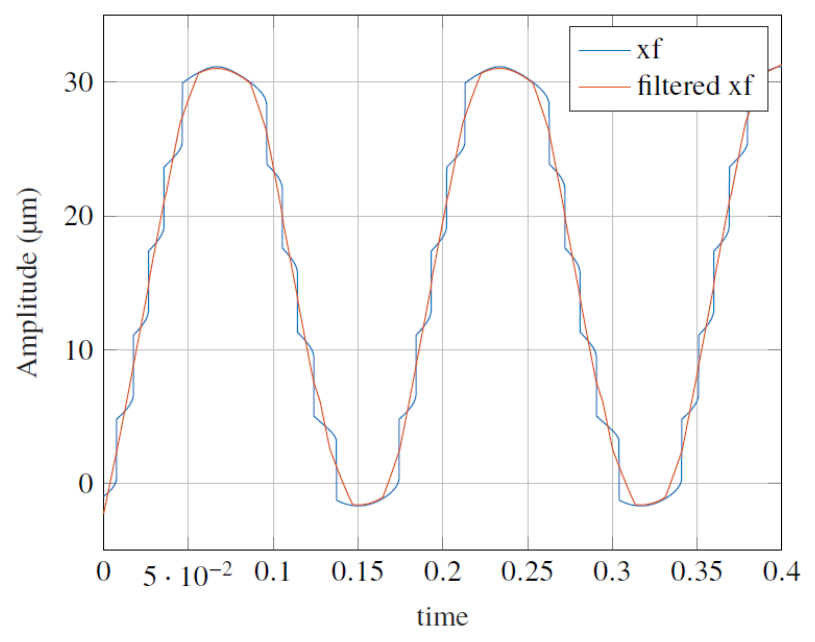

Fig. 9. Plot of perturbed phase $x f$ (blue) and corresponding filtered (Savitzky-Golay) form(red) for $\mathrm{C}=4, \alpha=5$

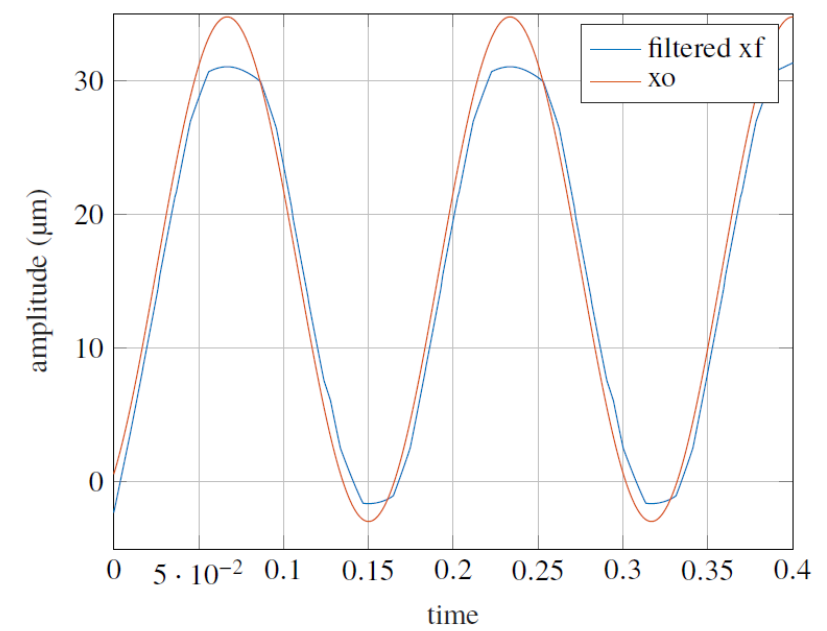

Fig. 10. Comparison of filtered (Savitzky-Golay) perturbed phase $x_{f}$ (blue) and reference $x_{o}(\mathrm{red})$ for a displacement of $6 \mathrm{kHz}$ with mean absolute error of $1.9976 \mu m$ for $\mathrm{C}=4, \alpha=5$

transform of perturbed phase for $6 \mathrm{~Hz}$ displacement, $10 \mathrm{kHz}$ sampling frequency with $\mathrm{C}=4, \alpha=5$, acts as almost the same as Fourier transform of unperturbed phase but with new magnitudes added to frequencies where Fourier transform of unperturbed phase approaches zero. Moreover, top peaks difference is $1.09 \mu \mathrm{m}$, which implies suppositions over the spectrum of the unperturbed phase. A simple denoising process can take care of high-frequency magnitudes, as mentioned in TFSP method [11], carried out by a wavelet (Daubechies-4 wavelet) [4] at 7th level, creates an error of $4.5 \mu \mathrm{m}$, which is quite not enough, since our range is in $\mu \mathrm{m}$. This is shown in figure(8).

Another way to use the approach of exploiting the noisy nature of the perturbed phase is to use Savitzky-Golay filter [9] which is a smoothing filter that tends to fit polynomials on subsets of adjacent data points. Given the structure of the

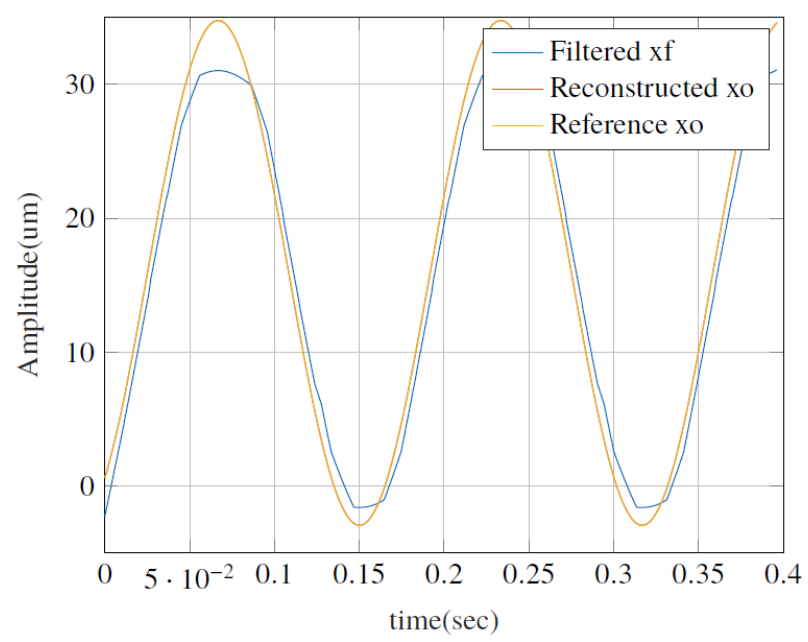

Fig. 11. Comparison of filtered (Savitzky-Golay) perturbed phase $x_{f}$ (blue) deformed into estimated $x_{o}$ (red) with reference $x_{o}$ (yellow) for a displacement of $6 \mathrm{kHz}$ with mean absolute error of $0.0148 \mu \mathrm{m}$ for $\mathrm{C}=4, \alpha=5$

\begin{tabular}{|c|c|}
\hline $\mathrm{C}(\alpha=5)$ & mean absolute error $(\mu \mathrm{m})$ \\
\hline \hline 1 & 0.064 \\
\hline 3 & 0.052 \\
\hline 5 & 0.079 \\
\hline 7 & 0.089 \\
\hline 9 & 0.027 \\
\hline
\end{tabular}

TABLE I

COMPARISON OF MEAN ABSOLUTE ERRORS OF SINGLE FREQUENCY DISPLACEMENT FOR DIFFERENT VALUES OF C FOR FIXED $\alpha$ WHEN $\mathrm{C}$ IS UNKNOWN

perturbed phase, as shown in figure (6) as triangular structures over the unperturbed phase, makes Savitzky-Golay filter, a good way method of spectral filtering. As shown in figure (9) and (10), Savitzky-Golay filter is carried out, with a frame length of 201 points, for a displacement of $6 \mathrm{~Hz}$ sampled at $10 \mathrm{kHz}$. Fig (11), shows that error between reconstructed phase $\left(x_{o r}\right)$ and $x_{o}$ is bounded by $1.99 \mu \mathrm{m}$ which is still not sufficient and therefore requires additional treatment. We can make important deduction from figure (8) and (10) that $x_{\text {or }}$ acts as deformation of $x_{o}$ that can be continuously deformed onto $x_{o}$. Following further steps of the proposed algorithm, we get figure (11), with a good mean absolute error of $14.8 \mathrm{~nm}$. Employing the algorithm, we get the following table which represents mean absolute error for mentioned algorithm for reconstructed unperturbed phase $x_{o}$ for $6 \mathrm{~Hz}$ displacement, covering all feedback regimes $(\mathrm{C}=1$ to 9 with 2 units spacing). Similarly,following table represents mean absolute error for reconstructed unperturbed phase for displacement having of 6,12 and $18 \mathrm{~Hz}$ frequency components covering all feedback regimes.

1) Remark: Mean of errors for displacement with one frequency component is $62.2 \mathrm{~nm}$, while for three frequency components is $293 \mathrm{~nm}$, while covering all feedback regimes.It can be observed that as the bandwidth of the displacement(and thus unperturbed phase as it is just a scalar mapping of displacement) increases, accuracy of proposed algorithm de- 


\begin{tabular}{|c|c|}
\hline $\mathrm{C}(\alpha=5)$ & mean absolute error $(\mu \mathrm{m})$ \\
\hline \hline 1 & 0.168 \\
\hline 3 & 0.114 \\
\hline 5 & 0.257 \\
\hline 7 & 0.418 \\
\hline 9 & 0.512 \\
\hline \multicolumn{2}{|c|}{ TABLE II }
\end{tabular}

COMPARISON OF MEAN ABSOLUTE ERRORS OF MULTI FREQUENCY DISPLACEMENT FOR DIFFERENT VALUES OF C FOR FIXED $\alpha$ WHEN C IS UNKNOWN

creases.

\section{CONCLUSION}

It has been proven that a strategy for estimating displacement from perturbed phase $x_{f}$ can not be a method involving solely $x_{f}$ as a continuous function of $x_{o}$ or through displacement method. Moreover, the subset of such a strategy is that the nature of the practical action of deriving $x_{o}$ from $x_{f}$ spectral filtering process followed by some perturbative or homotopical deformation. This strategy can be adopted when parameters are uncertain and displacement to be determined when we get SM signal (and then perturbed phase through methods like phase unwrapping method [11]). A less computationally intensive approach on this strategy has been employed, where the proposed algorithm produced a mean error of $62.2 \mathrm{~nm}$, while considering all feedback regimes unbiasedly, when ' $\mathrm{C}$ ' is unknown.

\section{DECLARATIONS}

\section{AVAILABILITY OF DATA MATERIAL}

No.

\section{FUNDING}

This study was not funded.

\section{COMPETING INTERESTS}

The authors declare no conflicts of interest.

\section{AUTHOR'S CONTRIBUTIONS}

This paper has only one author.

\section{ACKNOWLEDGEMENTS}

I acknowledge Dr. Usman Zabit (National University of Sciences and Technology,Pakistan) for providing be simualation data.

\section{REFERENCES}

\section{REFERENCES}

[1] O. D. Bernal, U. Zabit, and T. Bosch. Classification of laser selfmixing interferometric signal under moderate feedback. Applied optics, 53(4):702-708, 2014.

[2] C. Bes, G. Plantier, and T. Bosch. Displacement measurements using a self-mixing laser diode under moderate feedback. IEEE transactions on instrumentation and measurement, 55(4):1101-1105, 2006.

[3] S. Donati. Developing self-mixing interferometry for instrumentation and measurements. Laser \& Photonics Reviews, 6(3):393-417, 2012.
[4] S. Golestan, M. Ramezani, J. M. Guerrero, F. D. Freijedo, and M. Monfared. Moving average filter based phase-locked loops: Performance analysis and design guidelines. IEEE Transactions on Power Electronics, 29(6):2750-2763, 2013.

[5] M. Krepela. Convolution inequalities in weighted lorentz spaces. Math Inequal. Appl, 17(4):1201-1223, 2014.

[6] R. Lang and K. Kobayashi. External optical feedback effects on semiconductor injection laser properties. IEEE journal of Quantum Electronics, 16(3):347-355, 1980.

[7] J. M. Nester. Invariant derivation of the euler-lagrange equation. Journal of Physics A: Mathematical, Nuclear and General, 21(21):L1013L1017, 1988.

[8] G. Plantier, C. Bes, and T. Bosch. Behavioral model of a self-mixing laser diode sensor. IEEE Journal of Quantum Electronics, 41(9):11571167, 2005.

[9] R. W. Schafer. What is a savitzky-golay filter?[lecture notes]. IEEE Signal processing magazine, 28(4):111-117, 2011.

[10] T. Taimre, M. Nikolić, K. Bertling, Y. L. Lim, T. Bosch, and A. D. Rakić Laser feedback interferometry: a tutorial on the self-mixing effect for coherent sensing. Advances in Optics and Photonics, 7(3):570-631, 2015.

[11] U. Zabit, O. D. Bernal, S. Amin, M. F. Qureshi, A. H. Khawaja, and T. Bosch. Spectral processing of self-mixing interferometric signal phase for improved vibration sensing under weak-and moderate-feedback regime. IEEE Sensors Journal, 19(23):11151-11158, 2019. 